\title{
Further evidence in favor of prior entry from endogenous attention to a location in space
}

\author{
Ralph S. Redden ${ }^{1}$ • Ghislain d'Entremont ${ }^{1}$ - Raymond M. Klein $^{1}$
}

Published online: 9 March 2017

(C) The Psychonomic Society, Inc. 2017

\begin{abstract}
Titchener's (1908) law of prior entry states that "the object of attention comes to consciousness more quickly than the objects which we are not attending to," or otherwise, that attended stimuli are perceived earlier than unattended stimuli. Shore, Spence, and Klein (Psychological Science, 12, 205212. doi:10.1111/1467-9280.00337, 2001) showed that endogenous visuospatial orienting does in fact elicit priorentry effects, albeit to a smaller degree than does exogenous visuospatial orienting. In disagreement with this finding, Schneider and Bavelier (Cognitive Psychology, 47, 333-366. doi:10.1016/S0010-0285(03)00035-5, 2003) found no effect of their instruction to attend. They concluded that nonattentional effects could masquerade as prior entry, which could account for findings such as those in Shore et al.'s endogenous condition. We investigated this empirical and theoretical discord by replicating the temporal-order judgment task used by Shore, Spence, and Klein, while manipulating and measuring endogenous orienting by way of an orthogonal color probe task. We showed evidence of prior entry as a consequence of endogenous orienting, supporting the conclusions of Shore, Spence, and Klein.
\end{abstract}

Keywords Prior entry · Endogenous orienting •

Temporal-order judgment $\cdot$ Color wheel $\cdot$ Bayesian analysis

Is the speed at which a stimulus is perceived affected by attention? This question has been asked for generations in the

Ralph S. Redden

rredden@dal.ca

1 Department of Psychology \& Neuroscience, Dalhousie University, 1355 Oxford St., 3rd Floor LSC, Halifax, Nova Scotia B3H 4R2, Canada field of experimental psychology (for reviews, see Spence, Shore, \& Klein, 2001, and Spence \& Parise, 2010). Titchener's fourth law of attention - the law of prior entry (1908, p. 251) —states that "the object of attention comes to consciousness more quickly than the objects which we are not attending to," or otherwise that attended stimuli are perceived prior to unattended stimuli. Since the turn of the century, though, evidence has been obtained both in support of and against the "prior-entry" effect (Redden, d'Entremont, \& Klein, 2017; Schneider \& Bavelier, 2003; Shore \& Spence, 2005; Shore, Spence, \& Klein, 2001; Spence et al., 2001; Vibell, Klinge, Zampini, Spence, \& Nobre, 2007), under conditions of both exogenous (or reflexive) and endogenous (or volitional) orienting. The purpose of this investigation was to determine whether the endogenous prior-entry effect reported by Shore et al. is robust against some minor methodological variations, or whether the claim made by Schneider and Bavelier that endogenous orienting does not elicit prior entry is supported. Before positioning our intent within the context of this debate in the literature, a typical task used to investigate this phenomenon will be described.

\section{The model task}

Prior entry is typically studied using a temporal-order judgment (TOJ) task. Participants are presented with a pair of stimuli that vary in their onset times (i.e., in stimulus onset asynchrony [SOA]). Some experimental manipulation is employed to align attention with one of these two stimuli. This experimental manipulation could be a transient event (to elicit exogenous attentional mechanisms) or a learned contingency (to elicit endogenous attentional mechanisms). The participant must then judge the order of the two stimuli. The data are typically summarized as psychometric functions in 
which the proportion of responses selecting one of the two stimuli is plotted as a function of temporal asynchrony. The point of subjective simultaneity (PSS) - the SOA at which the proportion of selecting each stimulus is .50-is the key indicator of whether prior entry has been elicited. The PSS represents the amount of temporal asynchrony required between the presentations of the stimuli for the participant to perceive them as having appeared simultaneously. A shift in PSS associated with an attentional manipulation-whereby an unattended stimulus needs to lead an attended stimulus for them to appear simultaneous - is attributed to prior entry affecting the speed of perceptual processing.

\section{Evidence for and against prior entry from endogenous visuospatial orienting}

Endogenous visuospatial orienting was shown to elicit prior entry by Shore, Spence, and Klein (2001). In their task, observers were required to report the temporal order of a vertical and a horizontal line. The lines could be presented in either the same or opposite peripheral placeholder box. A centrally presented arrow reliably predicted - on trials when the lines appeared in the same box (unilaterally) - in which box the stimuli would appear. These trials were methodological in nature and not analytic; only trials in which stimuli were presented one in each box (bilaterally) were analyzed. On a small subset of trials, rather than the TOJ stimuli, a probe was presented in one of the two placeholder boxes requiring a speeded detection response. Reaction times were faster for probes presented at the location indicated by the arrow, thus affirming that their endogenous-orienting contingency was successful. Importantly, asking observers to report the temporal order of the stimuli according to the orthogonal orientation rather than the location at which the first stimulus appeared eliminated the possibility of a first-order response bias, whereby observers might have been biased to report the side they were attending (Frey, 1990; Spence et al., 2001). Additionally, observers were asked to report either "Which First?" or "Which Second?"again an orthogonal decision relative to the spatial attentional manipulation. This between-subjects manipulation was also used as a means to control for and assess response bias, because second-order response biases may in fact affect responding such that when observers are unsure, they may be biased to select the stimulus on the attended side. Since such a response bias would have shifted the PSS in the opposite direction for "which second?" relative to "which first?" responses, the average of the two manipulations provided a measure of prior entry that controlled for response bias. Accordingly, as a result of the contingency associated with the arrow, Shore et al observed a 17-ms shift in the PSS for stimuli presented at the attended location, suggesting that endogenous visuospatial orienting does elicit prior entry.
Contrary to the findings of Shore et al. (2001), Schneider and Bavelier (2003) argued that endogenous visuospatial orienting does not, in fact, elicit prior entry, and instead they suggested that nonattentional mechanisms - such as response biases or sensory facilitation - could account for endogenouslike prior-entry effects. A key distinction, though, is that unlike Shore, Spence, and Klein, Schneider and Bavelier did not affirm that endogenous orienting had occurred in their task. A more recent investigation by Redden, d'Entremont, and Klein (2017) provides some support for Schneider and Bavelier's conclusions that endogenous visuospatial orienting might not elicit prior entry-albeit in a more real-world TOJ scenario, in which observers were required to make "safe" or "out" judgments at close first-base baseball plays. Redden et al. found no evidence that prior entry affected the proportions of "safe" versus "out" judgments in this real-world context. Additionally, a strength of their design relative to Schneider and Bavelier's was that Redden et al. affirmed that attention had been successfully oriented by way of an orthogonal attentional diagnostic, which we will describe next.

\section{Spatial contingency and measuring endogenous visuospatial orienting}

In the real-world context investigated by Redden, d'Entremont, and Klein (2017), observers had their attention endogenously biased to either the glove or the base between blocks by way of an orthogonal color probe task. Within a block, a color probe would appear on a subset of trials more often at either the base or the glove within the scene of a video of a close play at first base. On these trials, observers were required to identify the color of the probe rather than make a TOJ response. This orienting diagnostic, pioneered by Prinzmetal, Amiri, Allen, and Edwards (1998), is a novel approach to assessing the implications for information processing as a consequence of attention. In this method, participants are required to make color judgments on a full-spectrum color wheel-a method that affords a continuous measure of error. Zhang and Luck's (2008) discrete, fixed-resolution representation theory of visual working memory was developed using this task, which models two components of behavior that may contribute to performance: capacity and resolution. Lawrence (2010) advanced this theory computationally, reinforcing the ascription of capacity as the probability of encoding a stimulus, and resolution as the fidelity of encoding, given that a stimulus has been encoded.

In the present experiment, we explored whether endogenous orienting of attention does elicit prior entry, while using the enriched attentional diagnostic from Redden, d'Entremont, and Klein (2017). Again, Shore, Spence, and Klein (2001) showed an effect of prior entry in their endogenous-orienting task. 
Furthermore, their attentional-cueing manipulation was affirmed by way of a simple RT probe response showing faster probe RTs at cued than at uncued locations. Redden et al. showed no effect of prior entry in their endogenous-orienting task; however, like Shore et al, they also affirmed that attention had been successfully oriented by an attentional diagnostic. Herein lay the distinction we wished to evaluate: a key difference between Shore et al.'s and Redden et al.'s studies is that the former employed an endogenous cue that generated a contingency within the TOJ task, if, on a given trial, the TOJ stimuli were to be presented unilaterally, these stimuli would appear reliably at the location indicated by the centrally presented arrow cue. However the diagnostic probe had no contingency. This was not the case in the Redden et al. design. Here, endogenous orienting was elicited and measured by way of a contingency orthogonal to the TOJ task - the diagnostic probe was more likely to occur at one location than another, whereas the TOJ task had no contingency.

\section{The present study}

The goal of this investigation was to evaluate this nuanced distinction between the two experiments. We used the same TOJ task that was used in Shore, Spence, and Klein (2001), while employing the same spatial contingency/diagnostic as in Redden, d'Entremont, and Klein (2017). As had Shore et al., we required observers to make TOJs about the relative onsets of a vertical and a horizontal line in opposite placeholder boxes; however, we engendered an endogenous shift of attention by way of the same orthogonal color probe task used by Redden et al., albeit with minor modifications. In Redden et al.'s work, the probe was presented for $350 \mathrm{~ms}$ in a dynamic visual scene, and observers showed a very high probability of encoding $(M=$ 98.2\%). Due to the pragmatic concern of a ceiling effect on the probability of encoding when employing a visually homogeneous display, we manipulated color probe duration between subjects. This manipulation was entirely practically motivated, and as such we had no a priori, theoretically motivated predictions for how this factor might modulate behavior.

We predicted that spatial contingency would produce an effect on color wheel performance, whereby color probe performance would be better for probes presented at the more likely location. Contingent upon this outcome, should we see an effect on TOJs as a function of endogenous orienting, this would support the findings of Shore et al. (2001), but also affirm that prior entry can be manifest in contexts in which a spatial contingency is generated orthogonally to the TOJ task. Contrarily, should no effect of endogenous orienting be apparent on TOJs (Redden et al., 2017; Schneider \& Bavelier, 2003), two further explanations for the outcome would be possible: (1) Endogenous attention does not elicit prior entry, or (2) endogenous attention elicits prior entry only when the spatial contingency is within the TOJ task.

\section{Method}

Participants Forty people participated in the study; 29 of them were females, and 11 were males. The median age was 21 years. Participants received financial compensation (\$6/ $30 \mathrm{~min}$ ) or class credits for their participation in the study and provided informed consent approved by the Research Ethics Board, Dalhousie University.

Apparatus Stimuli were presented on an iMac running OS X 10.11.4. Images were displayed on a 27 -in. monitor with a resolution of 2,560 $\times 1,440$ pixels. Participants sat approximately $57 \mathrm{~cm}$ from the screen. All stimuli were presented on a dark gray background. The color probes had a diameter of approximately $0.5^{\circ}$. The fixation stimulus was an asterisk of equal width and height $\left(1^{\circ}\right)$ presented at the midpoint of the two peripheral placeholder boxes. The boxes had equal sides of $4^{\circ}$ and were displayed $11.3^{\circ}$ to the left and right of the fixation stimulus. The horizontal and vertical lines had a length of $3^{\circ}$. The color wheel (see below) had a diameter of $10.6^{\circ}$, with an annulus width of $2.7^{\circ}$. Participants indicated their TOJ responses by pressing either the " 8 " or " 2 " key on a keyboard, to signal vertical or horizontal responses, respectively. Color wheel responses were recorded via a mouse click.

Procedure After initiating a trial by pressing the "space bar," the participant was presented with an asterisk centered on the screen, with a hollow white box on either side of it. After a random interval ranging from 1,395 to $1,830 \mathrm{~ms}$, a vertical or horizontal line was presented in either the left or the right box. The other line was presented in the opposite box after a given SOA (Fig. 1). Each participant was required to make one of two TOJ judgments: "which line appeared first?" or "which line appeared second?". TOJ judgment type was a factor counterbalanced between subjects, with the levels "which first" and "which second." On color wheel trials, the probe was presented at the time of the onset of the first stimulus. On these trials, participants were required to indicate the color of the disk by using a constant-luminance color wheel (Fig. 2), rather than making a TOJ response. The color wheel was randomly rotated from trial to trial so as to avoid response biases to the positions of certain colors with respect to the computer screen and/or the mouse cursor. Color wheel responses were recorded as angular deviations from the actual probe color. The probe was presented for either $50,75,100$, or $200 \mathrm{~ms}$. Probe duration was varied between subjects. Twenty participants were exposed to the 200 -ms probe duration, three participants were exposed to the $100-\mathrm{ms}$ duration, one participant was exposed to the 50-ms duration, and the remaining 16 participants were exposed to the 75-ms duration. A dichotomous, counterbalanced between-subjects factor was created 


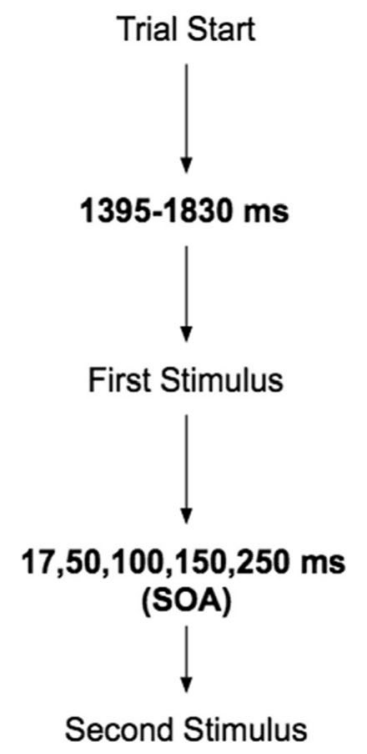

Fig. 1 Sequence of events in a trial. The trial starts with the presentation of an asterisk (center) flanked by white hollow boxes. After a random interval ranging from 1,395 to $1,830 \mathrm{~ms}$, the first stimulus was presented to either side (e.g., vertical line on the right). On color probe trials $(1 / 3)$, a colored disk appears at the same time as the first stimulus, on either side

with two levels: long probe duration ("long": $200 \mathrm{~ms}$ ) and short probe duration ("short": $100 \mathrm{~ms}$ or less).

Each participant completed three practice and two experimental blocks. The first block consisted of 40 practice TOJ trials. The second block consisted of 40 practice color wheel trials. In this block, attention was biased toward one of the two candidate placeholder locations by presenting probes at one of two locations (right or left) $80 \%$ of the time. The biased location alternated between participants so as to be counterbalanced. The third block was an experimental block (randomly intermixed color probe and TOJ trials) in which attention was biased toward the same location as in Block 2. The fourth block consisted of 40 practice color wheel trials, with attention biased to the location toward which attention had not been biased in the second and third block. The fifth block was an experimental

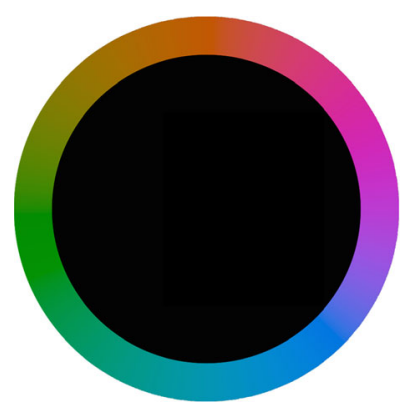

Fig. 2 Constant-luminance color wheel. Participants used a cursor to indicate the perceived color of the color probe

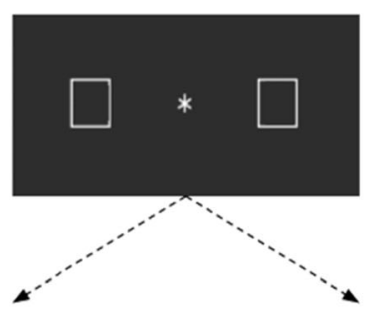

TOJ Trial $\quad$ Color Probe Trial

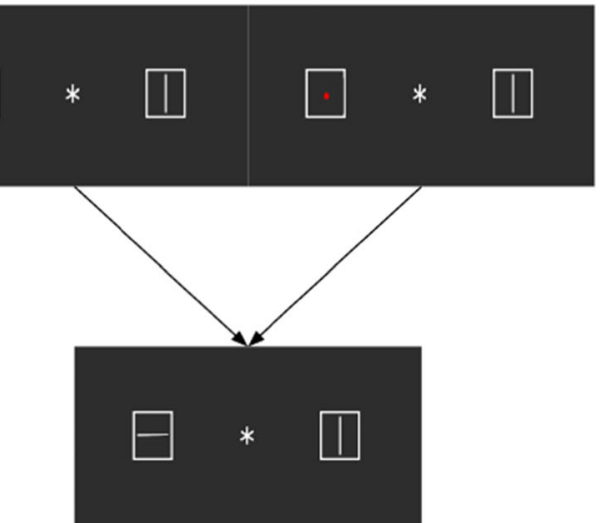

(e.g., red probe on the left). After one of five SOAs (17, 50, 100, 150, or $250 \mathrm{~ms}$ ), the second stimulus was presented in the opposite placeholder as the first stimulus (e.g., horizontal line on the left). All stimuli are presented on a dark gray background. The stimuli depicted here are not shown to scale

block (color probe and TOJ trials intermixed) in which attention was biased toward the same location as on Block 4. Both experimental blocks consisted of 240 trials, two thirds of which required TOJs, the other third of which required color wheel responses. The trial counts as a function of SOA for the experimental blocks are shown in Table 1. The practice blocks had proportionally identical SOA distributions.

Before starting the experiment, participants read a set of instructions (Appendix A) regarding all possible endogenous attention-orienting conditions. Furthermore, at the beginning of each block, participants received on-screen information regarding the endogenous attention-orienting condition of said block.

Analysis A mixture model was used to dichotomize color wheel performances into probability and fidelity of encoding (for a conceptual explanation of these parameters and a detailed description of the model, see Redden et al., 2017). The PSS and just-noticeable difference (JND) were defined as the mean and the standard deviation of a cumulative normal distribution. The data from both tasks (TOJ and color wheel) were evaluated in a joint model (mentioned below), which allowed for estimation of the correlation of the parameters between tasks.

A Bayesian hierarchical model was used for the statistical analysis, the motivation for which is also described in Redden, d'Entremont, and Klein (2017). A detailed description of this 
Table 1 Distribution of trials in a given block across all SOAs and both tasks (TOJ and Color Wheel)

\begin{tabular}{llllllllll}
\hline Trial Type & & & & & SOA & (ms) & & \\
-17 & -100 & -50 & -17 & 100 & 150 & 250 \\
\hline Color Wheel & 4 & 8 & 8 & 8 & 12 & 12 & 8 & 8 & 8 \\
TOJ & 8 & 16 & 16 & 16 & 24 & 24 & 16 & 16 & 16 \\
\hline
\end{tabular}

Negative SOAs indicate that the stimulus presented on the right preceded the stimulus presented on the left

model is found in Appendix B. Our parameter estimates are represented as posterior distributions (Fawcett, Lawrence, \& Taylor, 2016; Kruschke, 2014; Redden et al., 2017). Posteriors indicate the relative credibility of parameter values along a continuum of possible values after having accounted for one's empirically inspired prior beliefs and the present data (Kruschke, 2014). We will summarize the information contained in the posteriors by highest density intervals (HDIs). A 95\% HDI, for instance, covers the range of values within which we believe the true population parameter value falls with $95 \%$ probability. Ultimately, our model provided posterior distributions for each population parameter mean. We took the median value of these posterior distributions of means to be the point estimates for each parameter.

The model parameters themselves can be interpreted as standard-regression terms. We defined effects as the differences between two conditions. The model computed parameter estimates for probability in logit space and for fidelity and JND in logarithmic space, to facilitate the computation of population intercepts and effects. However, we will report back-transformed estimates for these parameters in order to facilitate interpretability. ${ }^{1}$

\section{Results}

\section{Summary of findings}

Psychometric functions (Fig. 3) show the intuitive expectations that when the presentation of the "left" substantially leads the "right" stimulus, the stimulus on the left will be selected as having appeared first with nearly perfect accuracy. Consistently, when the "right" substantially leads the "left" stimulus, the stimulus on the left is rarely (if ever) selected as having appeared first. Furthermore, as would be expected, these judgments become more difficult as the temporal asynchrony between these stimuli decreased. Most importantly, these judgments were affected by prior entry, whereby stimuli that were presented in the location compatible with the locus of attention were perceived as appearing earlier than the

\footnotetext{
${ }^{1}$ Transformed parameter estimates are reported in Appendix C, and descriptive statistics from the raw data-such as means, standard deviations, and correlations - are reported in Appendix D.
}

stimuli presented at the opposite location. Furthermore, attention was affirmed to be oriented by way of the orthogonal color probe task, which showed improved responses for probes presented at the more-probable location. Interestingly, it was also found that the duration of the diagnostic probe had implications for the nature of the effect of attention on color probe trials only, whereas prior entry was found irrespective of probe duration.

\section{Confirming locus of attention: Color wheel responses}

Probability of encoding The mean of the population intercepts of the probability of encoding was high $(\mathrm{Mdn}=.908$, $\left.\mathrm{HDI}_{95 \%}=.866, .946\right)$. We found a clear and substantial effect of probe duration (long minus short) on the probability of encoding $\left(\mathrm{Mdn}=.105, \mathrm{HDI}_{95 \%}=.034, .192\right)$, such that the

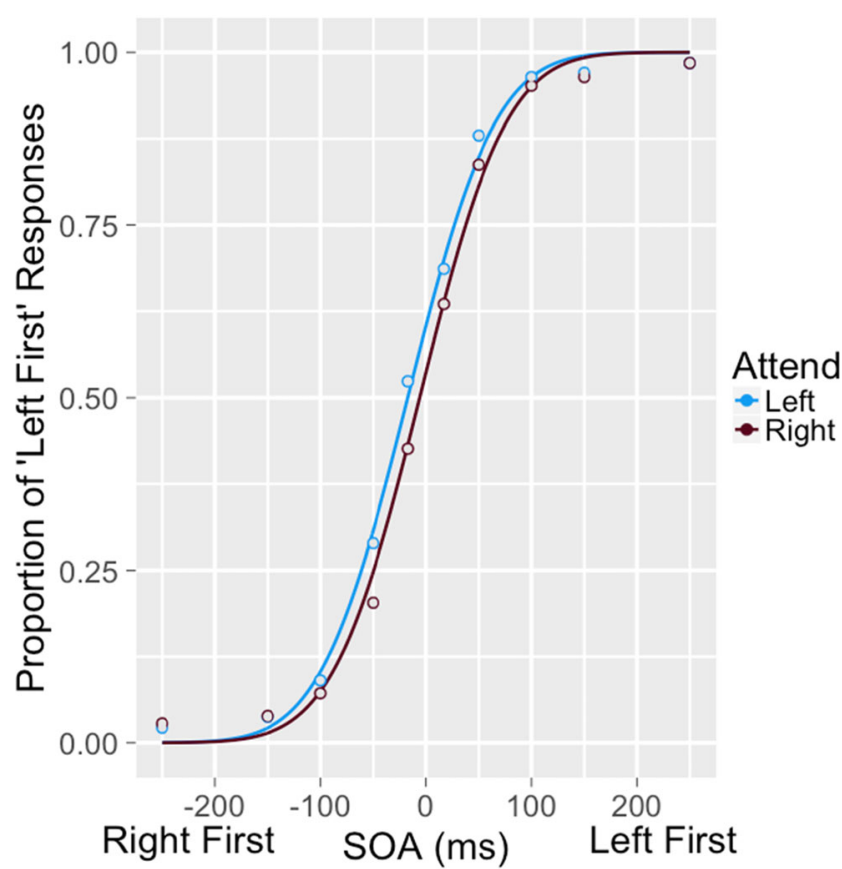

Fig. 3 Cumulative normal distributions for both attention conditions (attend right, red; attend left, blue), with the median of the posterior distribution of the point of subjective simultaneity (PSS) and the justnoticeable difference (JND) as the mean and standard deviation, respectively. Points with gray fill depict the raw data (proportions of "left first" responses by condition and SOA). Proportions of responses implying "left first" are plotted on the $y$-axis. Negative SOAs indicate that the right stimulus was presented first 


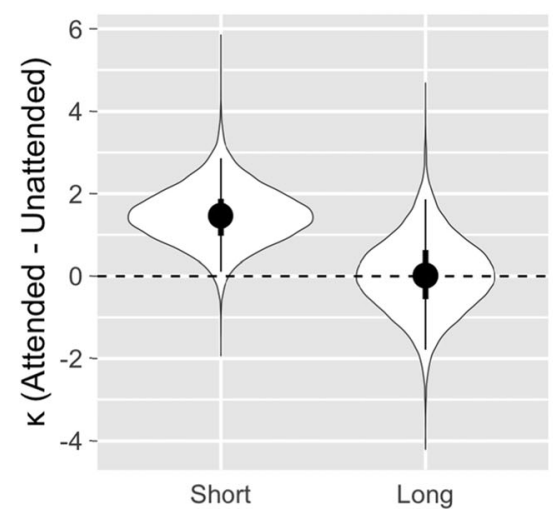

Fig. 4 Polygons depict the posterior credibility density distributions of the fidelity (left panel) and probability (right panel) of encoding-locus-ofattention effects (attended minus unattended) as a function of probe duration (short vs. long), with $\kappa$ (fidelity) and $\rho$ (probability) on the $y$-axis,

probability was about $10 \%$ higher for the long-duration than for the shorter-duration probes. Although there did not appear to be a main effect of locus of attention (attended minus unattended) on the probability of encoding ( $\mathrm{Mdn}=.011, \mathrm{HDI}_{95 \%}$ $=-.042, .069)$, the interaction between these variables trended, with a large point estimate of over $3 \%(\mathrm{Mdn}=$ $\left..033, \mathrm{HDI}_{95 \%}=-.078, .138\right)$. While the mean of the probability of encoding was especially high when the probe duration was long $\left(\mathrm{Mdn}=.962, \mathrm{HDI}_{95 \%}=.931, .985\right)$, the effect of locus of attention on the probability of encoding was notable. However, this effect was absent with short probe durations (Fig. 4, right panel).

Fidelity of encoding The posterior distribution for the mean of the population intercept of fidelity of encoding was centered at $6.24\left(\mathrm{HDI}_{95 \%}=5.45,7.04\right)$. As with effect of probe duration on probability, the effect on fidelity (long minus short) was large

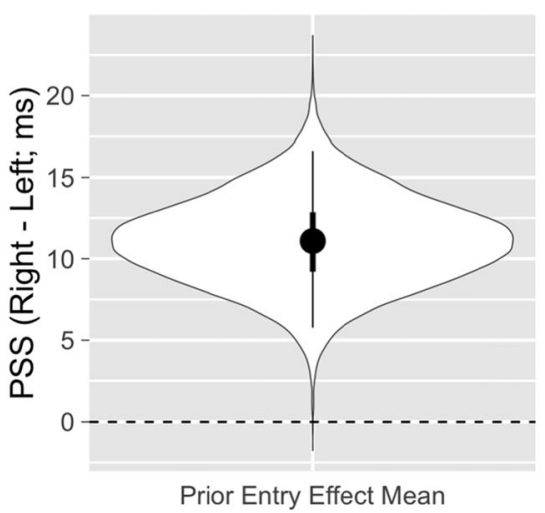

Fig. 5 Polygons depict the posterior credibility density distributions of the main effect of the locus of attention on the point of subjective simultaneity (PSS; left panel) and the effect of attention as a function of probe duration (right panel; short vs. long), with PSS (in milliseconds)

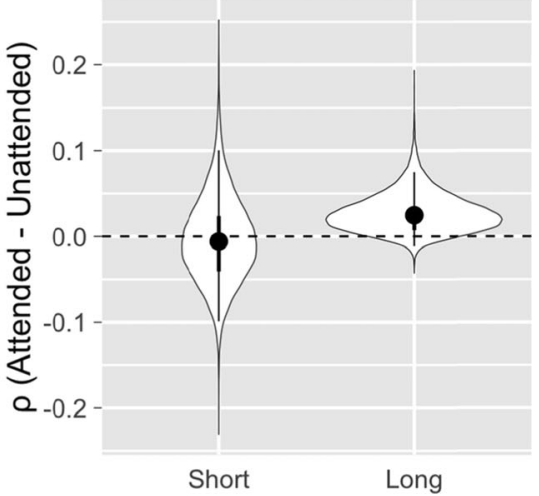

respectively. The dotted lines represent zero effects. The thick lines represent 50\% HDIs, whereas the thin lines represent 95\% HDIs. The large dot in each polygon depicts the median value of the distribution

$\left(\mathrm{Mdn}=2.56, \mathrm{HDI}_{95 \%}=1.01,4.12\right)$, such that fidelity was higher for the long than for the short probe duration probes. Although the main effect of locus of attention on fidelity trended positively $\left(\mathrm{Mdn}=0.73, \mathrm{HDI}_{95 \%}=-0.36,1.97\right)$, it was qualified by an interaction between these variables $\left(\mathrm{Mdn}=-1.45, \mathrm{HDI}_{95 \%}=-\right.$ $3.66,0.78$ ), in which the effect of locus of attention was present for the short probe durations, but absent when the probe was long. The direction of this interaction is illustrated in Fig. 4 (left panel). As can be seen in the figure, the results from the two dependent-variable encoding parameters suggest that the attentional manipulation was effective for both probe duration groups, but in different ways.

\section{Testing for prior entry: TOJs}

To conceptualize the meaning of the effect of locus of attention on PSS (the primary outcome) in the context of the TOJ task,

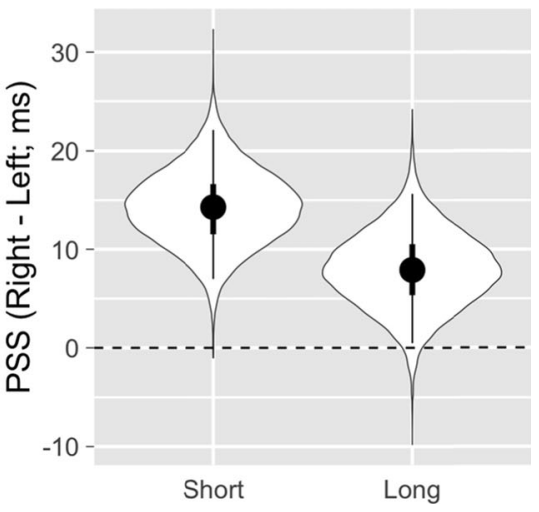

shown on the $y$-axes. The dotted lines represent zero effects. The thick lines represent the 50\% HDIs, whereas the thin lines represent 95\% HDIs. The large dot in each polygon depicts the median value of the distribution 
psychometric functions were generated for both locus-ofattention conditions (attend left and attend right; Fig. 4). The raw data (proportion of responses by condition and by SOA) are depicted as points to illustrate the degree to which the model fit the data. The means of the population intercepts of the PSS were centered at $-11.7 \mathrm{~ms}\left(\mathrm{HDI}_{95 \%}=-17.6,-6.0 \mathrm{~ms}\right)$, indicating an overall population-wise response bias toward the left location. The effect of the locus of attention on the PSS (attend right minus attend left) was exceptionally notable ( $\mathrm{Mdn}=$ $11.1 \mathrm{~ms}, \mathrm{HDI}_{95 \%}=5.8,16.6 \mathrm{~ms}$; Fig. 5, left panel). That is, we believe there was a near-95\% probability that the true population prior-entry effect exceeded $6 \mathrm{~ms}$, and over a $50 \%$ probability that it exceeded $11 \mathrm{~ms}$.

The effect of probe duration on the PSS (long minus short) trended positively ( $\mathrm{Mdn}=7.6 \mathrm{~ms}, \mathrm{HDI}_{95 \%}=-3.9,19.5 \mathrm{~ms}$ ). The interaction effect between these variables also showed a trend $\left(\mathrm{Mdn}=-6.4 \mathrm{~ms}, \mathrm{HDI}_{95 \%}=-16.8,4.3 \mathrm{~ms}\right.$ ), such that the effect of attention on the PSS was greater in the short than in the long probe duration condition (Fig. 5, right panel). Importantly, however, we observed prior-entry effects in both probe duration conditions.

The effect of judgment type on PSSs ("which second" minus "which first") was substantial ( $\mathrm{Mdn}=-14.2 \mathrm{~ms}$, $\mathrm{HDI}_{95 \%}=-26.0,-3.2 \mathrm{~ms}$ ): Participants were overall more likely to respond "left" when asked "which second" than they were when asked "which first." The interaction effect between judgment type and the locus of attention on PSSs trended such that the prior-entry effect was greater in the "which second" than in the "which first" condition, with a difference of $5.1 \mathrm{~ms}$ $\left(\mathrm{HDI}_{95 \%}=-5.5,15.6 \mathrm{~ms}\right)^{2}{ }^{2}$ Importantly, had the prior-entry effect been driven by response bias, the pattern in the "which second" condition would have been opposite that of the priorentry effect we observed in the "which first" condition. Since it was in the same direction, response bias is ruled out as a possible mechanism.

\section{Discussion}

The results in this experiment support the hypothesis that endogenous orienting does elicit prior entry. Stimuli were perceived as appearing earlier at attended locations, as evidenced by a shift in the population estimate of the PSS. Furthermore, we confirmed that attention was successfully manipulated, by way of a spatial contingency that showed enhanced encoding for probes presented at the more likely location. These findings support the conclusions of Shore, Spence, and Klein (2001) that endogenous visuospatial orienting can affect perceptual

\footnotetext{
${ }^{2}$ The results for JNDs are presented in Appendix E.
}

processes. We also believe that even though our estimate of endogenous prior entry was smaller than that of Shore et al., our investigation provides stronger evidence for this hypothesis, since it has been shown that arrow cues - like those used by Shore et al. - can also elicit exogenous attentional mechanisms (Hommel, Pratt, Colzato, \& Godijn, 2001; Ristic \& Kingstone, 2006). As such, Shore et al.'s estimate of endogenous prior entry may in fact be rendered from a combination of both endogenous and exogenous mechanisms. To our knowledge, we are the first to show that prior entry can be elicited from endogenous orienting elicited from a contingency orthogonal to the TOJ task. Many studies have shown evidence for prior entry resulting from endogenous orienting, in both unimodal and crossmodal tasks (Shore et al., 2001; Spence et al., 2001; Vibell et al., 2007; Yates \& Nicholls, 2009; Zampini et al., 2007; see also Stelmach \& Herdman, 1991); however, each of the aforementioned studies employed a contingency within the TOJ task in order to endogenously orient attention. This distinction more concretely supports the theory that prior entry is an attentional phenomenon, since it cannot be explained as either a by-product of experimental contingency or a response bias. Furthermore, these findings also support the robustness of the endogenous prior-entry effect across different shifts in endogenous attention, because Shore, Spence, and Klein (2001) showed prior entry in conditions under which endogenous attention was transiently deployed on a trial-by-trial basis as a result of an arrow contingency, whereas the present findings showed the effect when endogenous attention was stably deployed to a single location within a block as a result of the orthogonal color probe contingency.

We have shown the intuitive finding that performance on the color wheel task improves with a longer stimulus duration. However, we have also serendipitously discovered that the nature of the effect of attention on color wheel performance depends on probe duration. That is, when the probe duration is short, endogenous attention affects the fidelity of encoding the probe stimulus, but not the probability of encoding that stimulus. In contrast, when the probe duration is long, endogenous attention affects the probability of encoding the probe stimulus, but not its fidelity of encoding. Importantly, this double dissociation did not affect the presence of prior entry in the TOJ task, since highly credible PSS effects were observed for both probe durations. Zhang and Luck (2008) manipulated stimulus duration with masked stimuli and argued that stimulus duration affects probability but not fidelity of representation, suggesting an all-or-none process for the generation of durable visual representations 
that can survive the perception of new sensory inputs. The present investigation, however, showed effects of stimulus duration on both probability and fidelity of encoding. Several distinctions between these investigations may be the root of this difference. First, Zhang and Luck's conclusions were rendered from a design that did not manipulate visuospatial orienting. Second, the stimuli in the present design were not masked. Third, their stimulus duration manipulation was implemented within-subjects whereas the present manipulation was between-subjects. Future research ought to discern the degree that masking and stimulus duration may implicate the attentional consequences that affect encoding of visual inputs.

Although, as we noted above, the present investigation supports the hypothesis that endogenous visuospatial orienting does elicit prior entry, a question remains: How do we explain our previous failure to observe this effect in a more real-world context (Redden et al., 2017). As we previously noted, in both Redden, d'Entremont, and Klein (2017) and the present study, an endogenous attentional manipulation was successful. This was affirmed by way of improved color probe performance in both experiments for probes presented at the more likely location. However, the attentional effects on color probes differed between these experiments. Importantly, the TOJ task itself differed between the experiments: In the real-world experiment, observers were required to make judgments on the relative arrival times of two moving stimuli, whereas in the present study observers were required to temporally discriminate the times of occurrence of two onsets. Although this is certainly a relevant difference, we are not confident in coming up with an explanation for why comparison of the arrival times of moving objects might be more likely to escape an effect of attention than does the comparison of onset times. We recommend that further research be aimed at demonstrating the robustness of the different patterns of results.

The present investigation supports the hypothesis that endogenous visuospatial orienting can elicit prior entry, in accordance with the conclusions of Shore, Spence, and Klein (2001) and contrary to those of Schneider and Bavelier (2003). Furthermore, we have shown that the effect of endogenous attention on the probability and fidelity of encoding a stimulus depends on the signal strength of the target, such that endogenous orienting implicates the probability of encoding when signal strength is high, whereas endogenous orienting implicates the fidelity of encoding when signal strength is low. Importantly, prior entry was found in tandem with both encoding consequences.

Author note The present work was supported by a Natural Sciences and Engineering Research Council Discovery Grant awarded to R.M.K.

\section{Appendix A: Written instructions for participants prior to the experiment}

In this experiment, you will be presented with multiple trials, all of which will demand from you a response. You will initiate each trial on your own time. After initiating any given trial, you will be presented with an asterisk, centered on screen, with a hollow white box on both sides of it. After a short period of time, a vertical or horizontal line will appear in either the left or right box. Almost immediately after the presentation of this first line, the other line will appear in the opposite box. Under these conditions, you will be asked "which line appeared (FIRST/SECOND)?," and will be prompted to respond by clicking " 8 " on the key pad for "Vertical" or " 2 " on the key pad for "Horizontal." We will call this the "temporal order judgment" task. Alternatively, if a small, colored disk appears in the center of either box at the time that the first line appears on screen, then you will be asked to indicate the color of that disk using a full spectrum color wheel. We will call this the "color wheel" task. In both tasks, your goal is to respond as accurately as possible.

Now that you are familiar with the tasks at hand, let us summarize the order in which you will encounter these tasks. Trials will be broken down into blocks, of which there will be five in total. The first block will be a practice block consisting solely of temporal order judgment tasks, to familiarize you with that particular task. The second block will be a practice block consisting solely of color wheel tasks. Importantly, the colored disks will be more likely to appear on one side in this block. You will be told which side will be more likely at the beginning of the block. The third block will be an experimental block (i.e., pay attention, this one counts!) consisting of a mixture of both trials, which will be randomly ordered throughout the block. Crucially, the colored disks will be more likely to appear on the side that was the most likely in the second block. Again, you will reminded which side will be more likely at the beginning of the block. The fourth and the fifth blocks will be a replication of the second and third blocks, respectively, with the exception that the colored disk will be more likely to appear on the opposite side in these blocks.

\section{Appendix B: Bayesian hierarchical model details}

The model was implemented using RStan (Guo et al., 2016), the $\mathrm{R}$ interface for the Stan modeling language (Stan Development Team, 2015). Here we will simply give a highlevel summary of the model. The outcome variable for TOJ trials was a binary judgment ("vertical" or "horizontal") that was modeled with a Bernoulli distribution. The probability of a particular judgment followed a cumulative normal 
distribution with parameters JND (standard deviation) and PSS (mean). Each parameter was modelled as a function of participant-wise intercept and condition effect parameters. These parameters followed population distributions, which adaptively pooled information across participants. Relatively wide (i.e., less informed) priors were placed on the parameters of these population distributions. The outcome variable for colour wheel trials was response accuracy measured as angle deviation, which was modelled with the mixture model described in the methods section. The primary parameters, $\rho$ (probability) and $\kappa$ (fidelity), were also modelled hierarchically. Finally, the overall model incorporated a correlation matrix to quantify the linear relationship between population parameters. Ultimately, posterior credibility distributions were obtained for the PSS, JND, $\rho$, and $\kappa$ population intercept and attention effect means. The between-subjects effects of probe duration ("Short" vs. "Long"), along with their interactions with the within-subjects effects of attention, were estimated for all four parameters. The between-subjects effects of judgment type ("Which First?" vs. "Which Second?"), along with their interactions with the within-subject effects of attention, were only estimated for the PSS and JND. ${ }^{3}$

Model samples were drawn with a No-U-Turn Sampler, which is an extension of Hamiltonian Monte Carlo, a Markov chain Monte Carlo algorithm (Hoffman \& Gelman, 2014). Six chains were run, and each chain had 10,000 iterations and a "burn-in" period of 5,000. Therefore, we were left with 30,000 iterations. Model convergence was verified via a couple key diagnostics parameters (for all parameters, R-hat $\approx 1, N_{\text {Effective }}$ $>1,000$ ). Model validity was verified via posterior predictive checks whereby we evaluated whether the predictions from the posterior distribution of the model captured, within reason, the raw data from which the model was derived.

\section{Appendix C: 95\% HDIs and medians corresponding to posteriors for transformed values and all}

Median and 95\% HDI for the logarithm of fidelity of encoding

\begin{tabular}{lcc}
\hline Population Parameter (Means) & Median & $95 \%$ HDI \\
\hline Intercept & 1.80 & $1.67,1.93$ \\
Effect of Attention & 0.33 & $-0.35,0.95$ \\
Effect of Probe Duration & 0.43 & $0.19,0.69$ \\
Interaction Effect & -0.30 & $-0.67,0.06$ \\
\hline
\end{tabular}

\section{correlation coefficients}

${ }_{3}^{3}$ All code relating to the Bayesian analysis, including an explicit declaration of the model (e.g., priors), can be found online (http://or.psychology.dal.ca/ $\sim$ klein/dentremont/follow_up_toj_color.stan.zip).
Median and 95\% HDI for the log-odds of probability of encoding

\begin{tabular}{lll}
\hline Population Parameter (Means) & Median & 95\% HDI \\
\hline Intercept & 2.55 & $2.09,3.04$ \\
Effect of Attention & 0.33 & $-0.35,0.95$ \\
Effect of Probe Duration & 1.52 & $0.64,2.45$ \\
Interaction Effect & 0.77 & $-0.46,2.01$ \\
\hline
\end{tabular}

Median and 95\% HDI for the logarithm of JND

\begin{tabular}{lcl}
\hline Population Parameter (Means) & Median & 95\% HDI \\
\hline Intercept & -1.35 & $-1.50,-1.22$ \\
Effect of attention & -0.00 & $-0.11,0.11$ \\
Effect of probe duration & 0.02 & $-0.26,0.29$ \\
Attention $\times$ Probe Duration & 0.01 & $-0.22,0.23$ \\
Effect of Judgment Type & 0.07 & $-0.17,0.35$ \\
Probe Duration $\times$ Judgment Type & 0.06 & $-0.16,0.29$ \\
\hline
\end{tabular}

The logarithm of normalized JNDs (divided by 250) is presented here. All back-transformations reported in this article account for this normalization, which was meant to facilitate the sampling of the posterior distributions relating to the JND outcome

Correlations Correlations were computed on the transformed values (if applicable) of the model parameters, but for simplicity we refer to just the parameter names below, without specifying the calculation space. As can be seen in Fig. 6, the only notable correlations were between the effect of the locus of attention on PSS and the fidelity of encoding intercept, the probability of encoding intercept and the effect of attention on the probability of encoding, the probability intercept and the JND intercept, and the PSS and JND intercepts. For a priori reasons related to the hypothesis, we were interested in the relationship between effect of attention on either encoding parameter and the prior entry effect. These correlations both trended, albeit not convincingly (Fig. 6). 


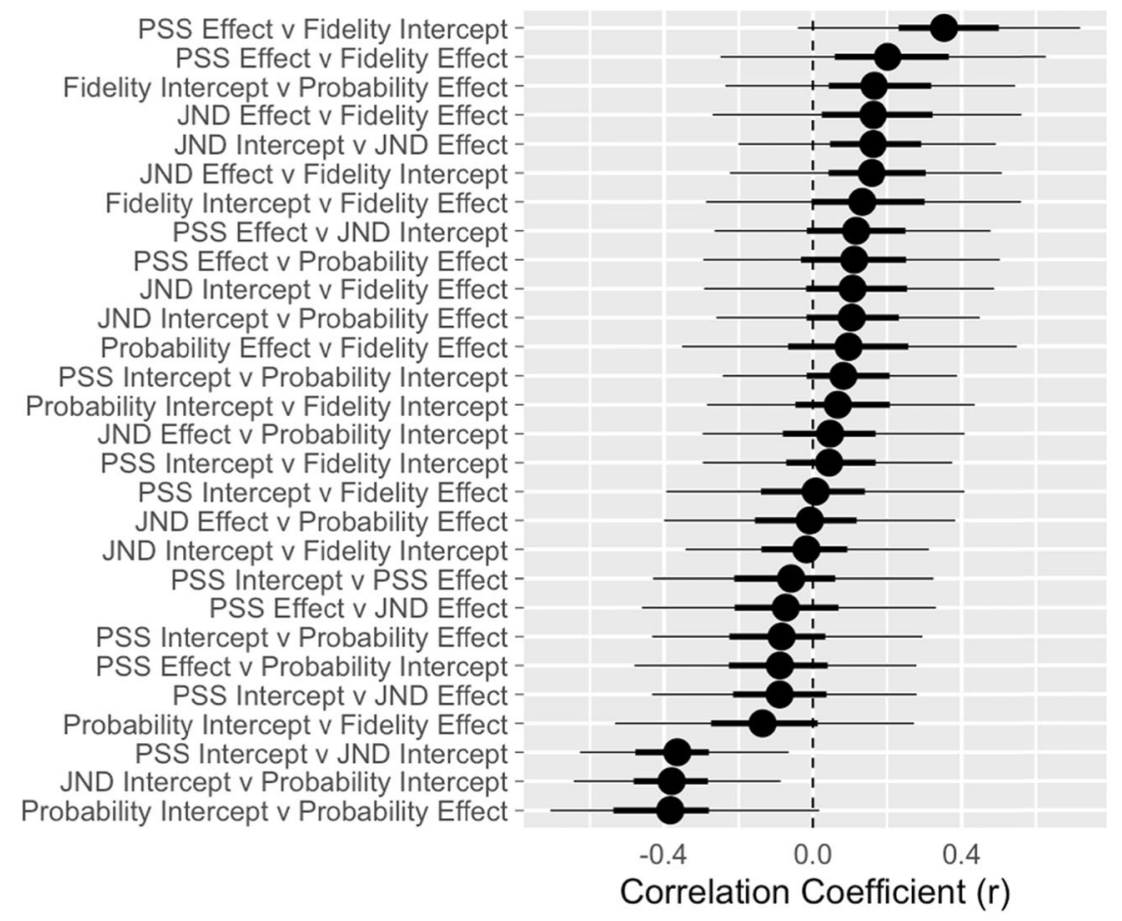

Fig. 6 Caterpillar plot of all possible correlation coefficients $(r)$. Each of the four outcome variables (just-noticeable difference [JND], point of subjective simultaneity [PSS], probability, and fidelity) has an intercept and a locus-of-attention effect parameter (means), meaning that there are eight parameters, and therefore 28 unique correlations. The parameters

that were computed in a transformed space are presented in that space (all but PSS intercept and effect). The dotted lines represent zero effects. The thick lines represent the 50\% HDIs, whereas the thin lines represent $95 \%$ HDIs. The large dots depict the median values of the distributions

\section{Appendix D: Descriptive statistics of the raw data}

Absolute color wheel error

\begin{tabular}{lllll}
\hline Attended & $\begin{array}{l}\text { Probe } \\
\text { Duration }\end{array}$ & $\begin{array}{l}\text { Absolute Color } \\
\text { Error (log deg) }\end{array}$ & $\begin{array}{l}\text { Naive Back- } \\
\text { Transformation } \\
\text { (deg) }\end{array}$ & $\begin{array}{l}\text { Absolute } \\
\text { Color Error } \\
\text { (deg) }\end{array}$ \\
\hline FALSE & FALSE & $3.58(0.42)$ & 35.8 & $38.9(16.0)$ \\
TRUE & FALSE & $3.42(0.32)$ & 30.5 & $32.0(10.6)$ \\
FALSE & TRUE & $3.14(0.42)$ & 23.2 & $25.7(14.9)$ \\
TRUE & TRUE & $2.94(0.23)$ & 18.8 & $19.3(4.5)$ \\
\hline
\end{tabular}

Fidelity of encoding

\begin{tabular}{lllll}
\hline Attended & $\begin{array}{l}\text { Probe } \\
\text { Duration }\end{array}$ & Log Kappa & $\begin{array}{l}\text { Naive Back- } \\
\text { Transformation } \\
\text { (Kappa) }\end{array}$ & Kappa \\
\hline FALSE & FALSE & $1.53(0.63)$ & 4.61 & $5.45(3.06)$ \\
TRUE & FALSE & $1.82(0.58)$ & 6.14 & $7.41(5.93)$ \\
FALSE & TRUE & $1.90(0.74)$ & 6.70 & $7.63(2.37)$ \\
TRUE & TRUE & $2.07(0.45)$ & 7.89 & $8.77(4.59)$ \\
\hline
\end{tabular}

Probability of e ncoding

\begin{tabular}{lllll}
\hline Attended & $\begin{array}{l}\text { Probe } \\
\text { Duration }\end{array}$ & Logit Rho & $\begin{array}{l}\text { Naive Back- } \\
\text { Transformation } \\
\text { (Rho) }\end{array}$ & Rho \\
\hline FALSE & FALSE & $2.47(2.12)$ & .922 & $.826(.201)$ \\
TRUE & FALSE & $1.88(1.20)$ & .867 & $.824(.149)$ \\
FALSE & TRUE & $3.06(1.36)$ & .955 & $.910(.113)$ \\
TRUE & TRUE & $3.96(1.31)$ & .981 & $.976(.034)$ \\
\hline
\end{tabular}

Just-noticeable difference (JND)

\begin{tabular}{llllll}
\hline Attended & $\begin{array}{l}\text { Probe } \\
\text { Duration }\end{array}$ & $\begin{array}{l}\text { Judgment } \\
\text { Type }\end{array}$ & $\begin{array}{l}\text { Log JND } \\
(\mathrm{ms})\end{array}$ & $\begin{array}{l}\text { Naive Back- } \\
\text { Transformation } \\
(\mathrm{ms})\end{array}$ & JND (ms) \\
\hline LEFT & FALSE & FIRST & $-1.45(0.49)$ & 58.8 & $66.1(38.4)$ \\
RIGHT & FALSE & FIRST & $-1.50(0.43)$ & 55.8 & $60.6(25.4)$ \\
LEFT & TRUE & FIRST & $-1.31(0.37)$ & 67.2 & $71.4(26.4)$ \\
RIGHT & TRUE & FIRST & $-1.36(0.32)$ & 64.2 & $67.0(19.3)$ \\
LEFT & FALSE & SECOND & $-1.28(0.48)$ & 69.5 & $77.2(37.9)$ \\
RIGHT & FALSE & SECOND & $-1.26(0.65)$ & 71.1 & $85.3(53.7)$ \\
LEFT & TRUE & SECOND & $-1.45(0.38)$ & 58.9 & $62.5(21.0)$ \\
RIGHT & TRUE & SECOND & $-1.44(0.52)$ & 59.5 & $66.4(29.5)$ \\
\hline & & & & &
\end{tabular}


Point of subjective simultaneity (PSS)

\begin{tabular}{lllr}
\hline Attended & Probe Duration & Judgment Type & \multicolumn{1}{c}{ PSS (ms) } \\
\hline LEFT & FALSE & FIRST & $-16.2(14.7)$ \\
RIGHT & FALSE & FIRST & $-4.4(15.5)$ \\
LEFT & TRUE & FIRST & $-6.1(27.3)$ \\
RIGHT & TRUE & FIRST & $6.7(21.0)$ \\
LEFT & FALSE & SECOND & $-30.2(16.0)$ \\
RIGHT & FALSE & SECOND & $-13.3(21.7)$ \\
LEFT & TRUE & SECOND & $-23.5(20.5)$ \\
RIGHT & TRUE & SECOND & $-14.7(19.6)$ \\
\hline
\end{tabular}

\begin{tabular}{|c|c|}
\hline Outcome Variables & Correlation \\
\hline JND intercept-PSS intercept & -.44 \\
\hline Probability intercept-PSS intercept & .03 \\
\hline Fidelity intercept-PSS intercept & .08 \\
\hline PSS effect-PSS intercept & -.02 \\
\hline JND effect-PSS intercept & -.12 \\
\hline Probability effect-PSS intercept & .01 \\
\hline Fidelity effect-PSS intercept & -.13 \\
\hline Probability intercept-JND intercept & -.44 \\
\hline Fidelity intercept-JND intercept & .01 \\
\hline PSS effect-JND intercept & .17 \\
\hline JND effect-JND intercept & .19 \\
\hline Probability effect-JND intercept & -.06 \\
\hline Fidelity effect-JND intercept & .21 \\
\hline Fidelity intercept-Probability intercept & -.08 \\
\hline PSS effect-Probability intercept & -.21 \\
\hline JND effect-Probability intercept & .13 \\
\hline Probability effect-Probability intercept & 0 \\
\hline Fidelity effect-Probability intercept & -.35 \\
\hline PSS effect-Fidelity intercept & .53 \\
\hline JND effect-Fidelity intercept & .19 \\
\hline Probability effect-Fidelity intercept & .29 \\
\hline Fidelity effect-Fidelity intercept & .44 \\
\hline JND effect-PSS effect & -.12 \\
\hline Probability effect-PSS effect & .1 \\
\hline Fidelity effect-PSS effect & .36 \\
\hline Probability effect-JND effect & -.08 \\
\hline Fidelity effect-JND effect & .28 \\
\hline Fidelity effect-Probability effect & -.11 \\
\hline
\end{tabular}

\section{Appendix E}

The mean of the population intercept of the JND was $65.0 \mathrm{~ms}$ $\left(\mathrm{HDI}_{95 \%}=56.1,74.5 \mathrm{~ms}\right)$. The effect of the locus of attention (attend right minus attend left) on the JND was centered near zero $\left(\mathrm{Mdn}=0.1 \mathrm{~ms}, \mathrm{HDI}_{95 \%}=-7.3,7.5 \mathrm{~ms}\right)$, as was the effect of probe duration (long minus short) on JND ( $\mathrm{Mdn}=1.0 \mathrm{~ms}$, $\left.\mathrm{HDI}_{95 \%}=-16.7,19.4 \mathrm{~ms}\right)$. Furthermore, the interaction effect between these variables appeared to be null $(\mathrm{Mdn}=0.4 \mathrm{~ms}$, $\mathrm{HDI}_{95 \%}=-14.5,14.9 \mathrm{~ms}$ ).

Similarly, the effect of judgment type (which second minus which first) on JNDs, and the interaction effect between this between-subjects variable and attention on JNDs seemed negligible $\left(\mathrm{Mdn}=4.8 \mathrm{~ms}, \mathrm{HDI}_{95 \%}=-11.3,22.3 \mathrm{~ms}, \mathrm{Mdn}=4.2\right.$, $\mathrm{HDI}_{95 \%}=-10.1,19.2 \mathrm{~ms}$, respectively).

\section{References}

Fawcett, J. M., Lawrence, M. A., \& Taylor, T. L. (2016). The representational consequences of intentional forgetting: Impairments to both the probability and fidelity of long-term memory. Journal of Experimental Psychology: General, 145, 56-81. doi:10.1037/xge0000128

Frey, R. D. (1990). Selective attention, event perception and the criterion of acceptability principle: Evidence supporting and rejecting the doctrine of prior entry. Human Movement Science, 9, 481-530.

Guo, J., Lee, D., Sakrejda, K., Gabry, J., Goodrich, B., de Guzman, J., . . . Fletcher, J. (2016). rstan: R interface to Stan. Retrieved from https:// cran.r-project.org/web/packages/rstan/index.html

Hoffman, M. D., \& Gelman, A. (2014). The No-U-turn sampler: adaptively setting path lengths in Hamiltonian Monte Carlo. Journal of Machine Learning Research, 15, 1593-1623.

Hommel, B., Pratt, J., Colzato, L., \& Godijn, R. (2001). Symbolic control of visual attention. Psychological Science, 12, 360-365.

Kruschke, J. K. (2014). Doing Bayesian data analysis: A tutorial with $R$, $J A G S$, and Stan (2nd ed.). Boston, MA: Academic Press.

Lawrence, M. A. (2010). Estimating the probability and fidelity of memory. Behavior Research Methods, 42, 957-968. doi:10.3758/BRM.42.4.957

Prinzmetal, W., Amiri, H., Allen, K., \& Edwards, T. (1998). Phenomenology of attention: I. Color, location, orientation, and spatial frequency. Journal of Experimental Psychology: Human Perception and Performance, 24, 261-282. doi:10.1037/0096-1523.24.1.261

Redden, R. S., d'Entremont, G., \& Klein, R. M. (2017). Safe or out: Does the location of attention affect judgments at first base in baseball? Canadian Journal of Experimental Psychology. doi:10.1037/ cep0000118. Advance online publication.

Ristic, J., \& Kingstone, A. (2006). Attention to arrows: Pointing to a new direction. Quarterly Journal of Experimental Psychology, 59, 19211930. doi:10.1080/17470210500416367

Schneider, K. A., \& Bavelier, D. (2003). Components of visual prior entry. Cognitive Psychology, 47, 333-366. doi:10.1016/S00100285(03)00035-5

Shore, D. I., \& Spence, C. (2005). Prior entry. In L. Itti, G. Rees, \& J. Tsotsos (Eds.), Neurobiology of attention (pp. 89-95). Amsterdam, The Netherlands: Elsevier.

Shore, D. I., Spence, C., \& Klein, R. M. (2001). Visual prior entry. Psychological Science, 12, 205-212. doi:10.1111/1467-9280.00337

Spence, C., \& Parise, C. (2010). Prior-entry: A review. Consciousness and Cognition, 19, 364-379. doi:10.1016/j.concog.2009.12.001

Spence, C., Shore, D. I., \& Klein, R. M. (2001). Multisensory prior entry. Journal of Experimental Psychology: General, 130, 799-832. doi: 10.1037/0096-3445.130.4.799

Stan Development Team. (2015). Stan modeling language: User's guide and reference manual. Retrieved from http://mc-stan.org/

Stelmach, L. B., \& Herdman, C. M. (1991). Directed attention and perception of temporal order. Journal of Experimental 
Psychology: Human Perception and Performance, 17, 539550. doi:10.1037/0096-1523.17.2.539

Titchener, E. B. (1908). Lectures on the elementary psychology of feeling and attention. New York, NY: Macmillan.

Vibell, J., Klinge, C., Zampini, M., Spence, C., \& Nobre, A. C. (2007). Temporal order is coded temporally in the brain: Early event-related potential latency shifts underlying prior entry in a cross-modal temporal order judgment task. Journal of Cognitive Neuroscience, 19, 109-120.
Yates, M. J., \& Nicholls, M. E. R. (2009). Somatosensory prior entry. Attention, Perception, \& Psychophysics, 71, 847-859. doi:10.3758 APP.71.5.847

Zampini, M., Bird, K. S., Bentley, D. E., Watson, A., Barrett, G., Jones, A. K., \& Spence, C. (2007). "Prior entry" for pain: Attention speeds the perceptual processing of painful stimuli. Neuroscience Letters, 414, 75-79.

Zhang, W., \& Luck, S. J. (2008). Discrete fixed-resolution representations in visual working memory. Nature, 453, 233-235. doi: 10.1038 /nature 06860 